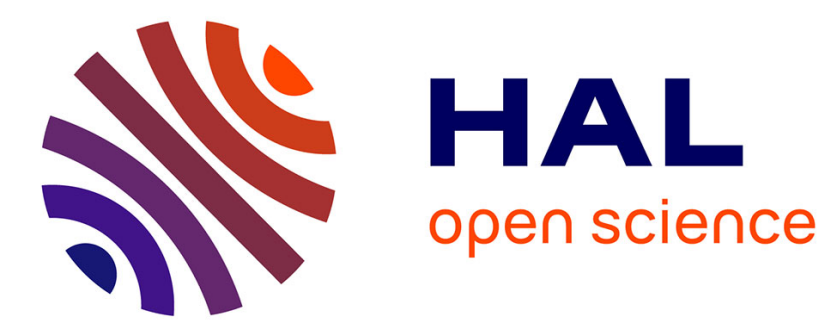

\title{
Applications of population approaches in toxicology
}

Frédéric Y. Bois

\section{To cite this version:}

Frédéric Y. Bois. Applications of population approaches in toxicology. Toxicology Letters, 2001, 120 (1-3), pp.385-394. 10.1016/S0378-4274(01)00270-3 . ineris-00961855

\section{HAL Id: ineris-00961855}

\section{https://hal-ineris.archives-ouvertes.fr/ineris-00961855}

Submitted on 20 Mar 2014

HAL is a multi-disciplinary open access archive for the deposit and dissemination of scientific research documents, whether they are published or not. The documents may come from teaching and research institutions in France or abroad, or from public or private research centers.
L'archive ouverte pluridisciplinaire $\mathbf{H A L}$, est destinée au dépôt et à la diffusion de documents scientifiques de niveau recherche, publiés ou non, émanant des établissements d'enseignement et de recherche français ou étrangers, des laboratoires publics ou privés. 


\title{
Applications of Population Approaches in Toxicology
}

\author{
FREDERIC Y. BOIS ${ }^{a}$ \\ $a$ INERIS, VERNEUIL EN HALATTE, FRANCE
}

\begin{abstract}
Many experimental or observational studies in toxicology are best analysed in a population framework. Recent examples include investigations of the extent and origin of intra-individual variability in toxicity studies, incorporation of genotypic information to address intra-individual variability, optimal design of experiments, and extension of toxicokinetic modelling to the analysis of biomarker studies in industrial hygiene or epidemiological contexts. Bayesian statistics provide powerful numerical methods for fitting population models, particularly when complex mechanistic toxicokinetic or toxicodynamic models are involved. Challenges and limitations to the use of population models, in terms of basic structure, computational burden, ease of implementation and data accessibility, are identified and discussed.
\end{abstract}

\section{KEYWORDS}

Bayes, Toxicodynamic models, Toxicokinetic models, Population Models.

\author{
Correspondence: Dr. Frédéric Y. Bois \\ Experimental Toxicology Laboratory \\ INERIS \\ Parc ALATA, BP 2 \\ F-60550 Verneuil en Halatte \\ France \\ +33 344556596 (phone) \\ +33 344556899 (fax) \\ Frederic.Bois@ineris.fr
}




\section{INTRODUCTION}

Uncertainty and variability affect potentially all experimental results. Both are prevalent when dealing with biological material or with human subjects. While uncertainty can be construed as a nuisance to Cartesian minds, variability has a different status. It may be necessary to assess both, for example when predicting risks. In such case, confidence in predictions and evaluation of the range of individual risks within a population are important considerations. Uncertainty is clearly different from variability:

- Uncertainty is essentially due to lack of knowledge (Rowe, 1994) and may have various sources. Measurements are made only with finite precision. When data are used to fit toxicokinetic (TK) or toxicodynamic (TD) models, there is always some "noise" about the resulting model parameters values. In addition, the fact that a population sample is studied also introduces an element of randomness. Simplifications or mispecifications of the fitted models also yield parameter uncertainties. Finally, extrapolations of in vitro results to in vivo, or across species, etc., are also a large source of variance.

- Variability refers typically to differences in characteristics among individuals (inter-individual variability), or across time within a given individual (inter-occasion, or intra-individual variability). It may stem from genetic differences, lifestyles, physiological status, age, etc. (Rowland and Tozer, 1989) . Experimental toxicology studies are in general specific to the (few) individuals or animal studied and extrapolating their results to larger and different populations requires careful consideration. TK studies for example are rarely about the toxicokinetics of a compound in a particular individual (clinical pharmacokinetics may be different in that respect). They rather aim at information about average toxicokinetics in sensitive populations, age groups, whole country population, etc., and at a quantification of the variability in those kinetics.

Uncertainty may be reduced by new experiments, the design of which can be optimised to bring a maximum of information (Beatty and Piegorsch, 1997), or by a better understanding of the biology and hence better models. This may take much efforts and decisions routinely need to be quickly made despite a fair amount of uncertainty about the situation examined. The "precautionary principle" is indeed an answer to the recognition of that need (Stirling, 1999) . Variability is inherent in animal and human populations. It cannot be reduced and seems even desirable for the stability of the global ecosystem (McCann, 2000). The fact that 
uncertainty is almost always present and hampers a precise assessment of variability, has led to the development of somewhat sophisticated statistical "population" models to disentangle them. A brief account of the development of those tools, of their basics, and a short survey of their burgeoning applications to toxicology form the topic of next sections.

\section{POPULATION MODELS}

Population models were originally introduced in the context of pharmacokinetic studies for drug development and evaluation (Beal and Sheiner, 1980; Beal and Sheiner, 1982; Sheiner, 1984) . A number of applications can be found in the pharmacokinetic literature (Aarons, 1992; Sheiner and Ludden, 1992; Vozeh et al., 1996; Yuh et al., 1994) . Until recently, little attention had been paid to these approaches in toxicokinetics. This is partly due to the fact that toxicokinetics used a lesser amount of human data, and focused on animal models. That picture is changing with the rapid development of human biomarker studies and improvements in analytical chemistry, which make is possible to follow in humans the kinetics of trace amounts of chemicals. Before our work in this area (Bois et al., 1996a; Gelman et al., 1996), the only published attempt to link population variability to TK modelling was presented by Droz et al. (1989), but no fitting to experimental data was performed in that exercise. Indeed, the scope of population models is not limited to pharmaco- or toxicokinetics. Dose-response studies, for example, can be fruitfully analysed with the same tools (Davidian and Giltinian, 1995; Palmer and Müller, 1998; Patron-Bizet et al., 1998; RacinePoon, 1985; Racine-Poon and Smith, 1990; Wakefield et al., 1994) .

The objective of population models is to extract, from data on individuals units (e.g., subject, family, group), a quantitative description of the variability of the kinetics of a compound within a large population. The basic idea is that the same model can describe the data for each unit, and that the model parameters differ randomly between units, or between occasions within the same unit, etc. Such randomness characterises variability per se, and is described by (multivariate) probability distributions. Individuals' renal clearance, for example, will be assumed to be normally distributed around a "population mean", with a "population variance" which measures variability in the population. Population means and variances (one for each kinetic parameter supposed to vary across units) are termed "population" parameters. They are estimated by data fitting, together with the model parameters for each unit. Other population parameters could be defined, such as parameter covariances (if some parameters are supposed to be correlated at the 
population level), or degrees of freedom if a (student) $t$-distribution is assumed instead of the normal distribution.

A typical population TK model is presented in Figure 1 (Bois et al., 1996a; Bois et al., 1996b; Gelman et al., 1996; Wakefield, 1996). The "hierarchical" or "multilevel" structure of the model is apparent, with two major components: the subject level and the population level.

At the subject level, data $(\mathbf{y})$ are measured at times $\mathbf{t}$, after exposure to dose $\mathbf{E}$. A set of individual characteristics (parameters, $\boldsymbol{\theta}$ ) also condition the observations. Some of these characteristics maybe known (e.g., sex, age, genotype), in which case they are considered as "covariates", but usually some interesting characteristics are unknown and need to be estimated from the data. That estimation can be achieved by data fitting. In Figure 1, unknown quantities are enclosed in circles and known quantities in square boxes. Data fitting requires basically the definition of two models:

- First, a statistical model should link some "predictable" aspects of the data (e.g., their expected values, or their variance) to the actual measurements (y). Deviations between measurements and their expected values may be due to limited assay precision, modelling error, or random intraindividual variability. Such deviations are typically assumed to be independent normal or lognormal random variables, with mean zero and variance $\sigma^{2}$. That variance can be set to a known value (e.g., if analytical precision has been independently assessed and if modelling error is negligible). It can also be estimated along with the other parameters. The model chosen will specify the data "likelihood". That likelihood is proportional to the probability of the observed data, given a model and its parameter values.

- Second, a TK or TD model, $f$, should link the chosen "predictable" to $\mathbf{E}$, $\mathbf{t}$, and $\boldsymbol{\theta}$, or any other relevant "explanatory" variable.

At the population level, inter-unit variability is described by considering that the individual parameters, $\theta$, are randomly drawn from given statistical distributions, each with population mean $\boldsymbol{\mu}$ and population variance $\Sigma^{2}$, as described above. Population means and variances are to be estimated together with the parameters $\boldsymbol{\theta}$ and the residual variance $\sigma^{2}$.

What about uncertainty in the above framework? Uncertainty enters in the fitting process in two ways: 
- First, through all variance sources aggregated into the residual variance $\sigma^{2}$ (typically, limited assay precision, modelling error, and un-modelled levels of variability). Note that it is possible to disaggregate $\sigma^{2}$ in order to identify separately its various components. For example, a further level in the hierarchy can be defined to deal with intra-unit variability (Lunn and Aarons, 1997) . In that case, residual variance can be reduced if intra-unit variability is significant. However, some measurement and modelling errors are likely to remain present, even with the best available data and models. A side-effect of that "noise" is to allow different model parameter values to yield adequate fits to the data (what is meant by adequate is determined by the statistical measurement model). That results in uncertainty about all the quantities estimated in the fitting process (population parameters, individual characteristics, etc., and even $\sigma^{2}$ if it is adjusted). This uncertainty may entangle estimates through fitting-induced correlations, which make it difficult to interpret the results when many parameters are adjusted.

- A less obvious source of uncertainty, which affects the population parameters, is the finite and often small size of the population sample studied. Even if the characteristics of each individual were perfectly known (from perfect data and models), the population parameter values inferred from a non-exhaustive sample would still be affected by uncertainty.

What are the advantages of population modelling? Most of all, it is a clean way to model levels of variability in data obtained on populations. The "naive" approach which consists in fitting individual data separately, collecting individual parameter estimates and forming their average etc., does not work if very precise parameter estimates cannot be obtained. In clinical pharmacokinetics and in toxicokinetics, the data on each individuals tend to be sparse, for ethical, feasibility or cost reasons. Such data usually lead to fairly uncertain parameter estimates and population approaches are then warranted. In addition, in a population model, information of each subject is reinforced by "borrowing strength" from the other subjects' data and the estimation process is improved overall. For example, when a subject's characteristic is barely estimable with its own data, the TK or TD link model can fail to give any reasonable estimate for the other characteristics (an effect of fitting-induced correlations, as in the flip-flop effect in pharmacokinetics). Discarding subjects data for that reason can make the experiment or trial worthless. In a population model, each subject's characteristics will tend to resemble that of the population (subjects are drawn toward the 
population mean), in particular if little data is available on that subject, and the estimation can proceed without information loss.

\section{BAYESIAN ANALYSIS OF POPULATION MODELS}

A number of methods have been proposed to fit models and estimate their parameters within a population framework. One way to classify them is into parametric and nonparametric methods. In the parametric approach, the shape of the population distribution is assumed to be known, and the population parameter values are quantities to estimate (Beal and Sheiner, 1982; Racine-Poon and Smith, 1990). That approach is the most often used. In the nonparametric case, no assumptions are made about the shape of the population distributions, and both shape and parameters are estimated from the data (Davidian and Gallant, 1992; Mallet et al., 1988).

Another classification of the relevant statistical methods differentiates maximum likelihood (Beal and Sheiner, 1980) and Bayesian approaches (Racine-Poon, 1985). Modelling in toxicology ought to be particularly well served by a Bayesian approach. TK/TD models tend to deal with scarcity of data and extrapolation needs (e.g., inter-species, inter-dose) by relying on complex mechanistic structures (Kohn, 1995) . Physiologically-based toxicokinetic (PBTK) models (Bois, 1999) or cancer models (Tan, 1991) can be fairly sophisticated, compared to regression or classical compartmental models. In a Bayesian framework, nonlinear models and non-standard statistical distributions are easily dealt with (Bernardo and Smith, 1994) . More importantly, a priori physiological information (such as found in the literature, in vitro, etc.) about parameters can be seamlessly incorporated into the data analysis (Gelman et al., 1996) . Finally, modern numerical techniques have made Bayesians unafraid of the many parameters of population models.

In a Bayesian setting, all unknowns, such as $\boldsymbol{\theta}, \boldsymbol{\mu}$ and $\Sigma^{2}$ in the above example (Figure 1), are considered as random variables. The probability distribution of an unknown parameter is interpreted in terms of degrees of belief about its possible values. Before analysing an experiment, a "prior" probability distribution, $\mathscr{P}$, is constructed for each unknown to reflect current knowledge about it. The use of a probability distribution to represent prior knowledge offers a unified way to account for precise as well as vague information available before an experiment. When little knowledge is available on a particular parameter, a non-informative distribution (i.e., flat-shaped) can be used. If stronger prior knowledge is at hand, the prior should represent that information as appropriately as possible. Note that information from the literature tends to relate to 
average values or variances for a population and is naturally handled in a population model. With a Bayesian approach, such information can be directly used under the form of prior distributions for population parameters such as $\boldsymbol{\mu}$ and $\Sigma^{2}$ (see above).

The set of prior distributions is then updated using the data values, $\mathbf{y}$, to yield a "posterior" probability distribution. While the prior distributions may be specified independently from each other, the posterior is a joint distribution of all unknowns (i.e., correlations induced by the model fitting process are accounted for). Bayes' theorem indicates that the posterior probability distribution of $\boldsymbol{\theta}, \boldsymbol{\mu}$ and $\Sigma^{2}$ given $\mathbf{y}$, noted $P\left(\boldsymbol{\theta}, \boldsymbol{\mu}, \Sigma^{2} \mid \mathbf{y}\right)$, is proportional to the product $\mathcal{P}$ of all priors by the data likelihood defined above, $P\left(\mathbf{y} \mid \boldsymbol{\theta}, \boldsymbol{\mu}, \Sigma^{2}\right)$ :

$$
P\left(\boldsymbol{\theta}, \boldsymbol{\mu}, \sum^{2} \mid \mathbf{y}\right) \propto \mathcal{P} \times P\left(\mathbf{y} \mid \boldsymbol{\theta}, \boldsymbol{\mu}, \Sigma^{2}\right)
$$

The hierarchical structure of a population model allows a splitting of the likelihood in two simpler terms:

$$
P\left(\boldsymbol{\theta}, \boldsymbol{\mu}, \sum^{2} \mid \mathbf{y}\right) \propto \mathcal{P} \times P(\mathbf{y} \mid \boldsymbol{\theta}) \times P\left(\boldsymbol{\theta} \mid \boldsymbol{\mu}, \sum^{2}\right)
$$

The exact form of term $P(\mathbf{y} \mid \boldsymbol{\theta})$ is dictated by the error model and the TK/TD model chosen. The form of $P\left(\theta \mid \boldsymbol{\mu}, \Sigma^{2}\right)$ depends on the distribution assumed for the individuals' characteristics within the population.

Bayes' rule can be viewed as a formula that shows how existing beliefs, formally expressed as probability distributions, should be modified by new information (Bernardo and Smith, 1994). Any statistical summary (mean, standard deviation, quantiles, correlations, marginal distribution, etc.) about the model parameters can be obtained from their posterior distribution. However, it is often difficult to obtain an analytical expression for $P\left(\boldsymbol{\theta}, \boldsymbol{\mu}, \Sigma^{2} \mid \mathbf{y}\right)$. Until recently, that has been a strong impediment to practical Bayesian analyses. Fortunately, the advent of Markov chain Monte Carlo (MCMC) methods has alleviated a number of these difficulties. These methods are powerful tools which can provide samples of parameter values out of their posterior distribution even without knowledge of an analytical expression for it (Gelman and Rubin, 1996; Gilks et al., 1996) . They have largely contributed to the recent proliferation of Bayesian analyses in applied statistics. 


\section{APPLICATIONS}

\section{TOXICOKINETICS}

Since population concepts were ported to toxicology via toxicokinetics, most applications seen so far fall into this section. TK models can be used to relate external exposures to toxicants to internal measures of dose. They typically describe the body as a set of compartments through which chemicals travel or are transformed. TK models fall into two main classes: "classical" models and physiologically-based (PBTK) models.

Classical TK models represent the body by several connected compartments (rarely more than three) within which chemicals are assumed to distribute homogeneously (Gibaldi and Perrier, 1982) . Compartments do not necessarily reflect the anatomy of the species of interest. The number of compartments and parameter values (e.g., exchange rates between compartments, volumes) are inferred from fitting the model to TK data. These models are thus commonly called empirical, or data-based. Population models can be used which such models as link functions between the data and individual characteristics, as is routinely done in pharmacokinetics.

In PBTK models, the body is subdivided into a series of anatomical or physiological compartments (Bailer, 1997) . Connections between compartments represent the blood or lymphatic circulation. PBTK models typically use three types of parameters: physiological parameters, such as breathing rate, blood flows and tissue volumes; physicochemical parameters, such as partition coefficients; and biochemical parameters, describing for example metabolic processes. The number of compartments depends on the objective of the study, and of the possible mode(s) of action and site(s) of toxicity of the chemical studied. The focus of PBTK modelling has been mainly on improving the scientific basis of animals to humans extrapolations (Gargas et al., 1995). The ability to tailor the parameter values of these models to a particular animal species offers, in theory, a firm basis for interspecies extrapolation. In fact, for most PBTK models, some parameters (in particular those describing metabolism) are difficult to extrapolate across species. A partial answers to this question may be provided by the "parallelogram approach" (Morris et al., 1996), but more often, some parameters values need to be obtained through model fitting to TK data. Obviously, that fitting should take into account the available physiological prior information on parameters. Prior information, as mentioned above, is usually about population means or variances, and population modelling offers a natural 
setting for inclusion of such information. To further complicate matters, PBTK models have many parameters (10 to 20 is typical) and are often nonlinear and without analytical solution. It is only recently that a general Bayesian method has been presented for fitting these models (Bois et al., 1996a; Gelman et al., 1996) . Before then, it was impossible to decide whether the fits were acceptable, the models reasonable, and what confidence to place in their results. A treatment of these models in a Bayesian population framework can therefore be advantageous, as further illustrated below, where we focus on specific study types.

\section{Applications to Experimental Studies}

\section{Animal Studies}

The analysis of animal experiments may benefit from the use of population models. Inter-animal variability in kinetics exists as well as in humans. It may not be so high because of the use of inbred strains, but is still there. Other levels of variability can also be present, such as inter-lot or inter-strain variability. If a large number of data points is gathered on each experimental unit and if characteristics of interest can be precisely assessed, a naive approach might be sufficient. However, when a PBTK model is used, the precision of estimates obtained is not guaranteed, given the number of parameters in those models. Using "standard" values for most physiological parameters and adjusting just a few "sweeps uncertainty under the rug" and leads to over-optimistic estimates of precision. A careful analysis would therefore proceed along the population modelling way, unless it can be proven that a simpler approach is also correct.

\section{Human Volunteers Studies}

Studies in human volunteers are being performed, despite potential ethical concerns, for several reasons; i. Modern analytical techniques allow the determination of kinetics after very low exposures (within environmental or occupational exposure ranges) posing minimal or zero risk to the subjects; ii. Animal studies may not be predictive of human responses; iii. Ethical concerns also exist for animal studies, even if rodents do not get to sit on ethics committees. Human toxicology studies are clearly geared toward risk assessment rather than toward research on mechanism of action, for example. In that context, an independent assessment of variability is a clear concern, and population modelling is a natural answer. PBTK models are here also in favour, since they can be used to simulate toxicokinetics under special conditions, such as physical activity (Dankovic and Bailer, 1994), or in specific targeted populations (e.g., 
pregnant women, children), by simply changing the values of some physiologic parameters. The same arguments as for animal experiments can be made in favour or the use of population models in that context. For further discussion, the reader is referred to our recent population TK analyses of volunteer exposures to tetrachloroethylene (Bois et al., 1996a; Gelman et al., 1996), benzene (Bois et al., 1996b) , trichloroethylene (Bois, 2000b; Bois, 2000a), or 1,3-butadiene (Bois et al., 1999). The inclusion of genotype information in such analyses is straightforward (Johanson et al., 1999), and should see considerable development in the near future.

\section{Optimal Experimental Design}

A useful extension of data analysis is the optimal design of experiments. Population modelling and Bayesian statistics can be jointly used to determine, for example, the optimal sampling times and optimal number of subjects to study (Atkinson et al., 1993; Merlé and Mentré, 1995; Wakefield, 1994) . In toxicokinetics, we recently used a classical two-compartment model to address similar questions for the planning of human volunteer exposures to 1,3-butadiene (Bois et al., 1999). We also recently established that numerical sampling techniques are powerful enough to allow the use of a PBTK model in the context of design optimisation (unpublished results).

\section{Applications to Biomarker Studies}

Biomarker studies of exposure (or effects) might well be the best field of application for population TK modelling. Multivariate endpoints are often measured and it makes sense to model their temporal correlations or autocorrelations via interpretable and knowledge-based TK models rather than through empirical covariance models. The small number of measurements usually made on individuals and the medium to large size populations investigated make them potential case-studies for the population approach. However, at least three reasons have slowed development in that area:

- First and foremost, biomarker studies (unless they use controlled exposure conditions, in which case they would fall under the "experiment" heading) have uncontrolled exposure conditions. It is possible, in a population statistical framework, to consider exposure as one of the parameters to estimate. This estimation challenge is known as the error-in-variables problem (Seber and Wild, 1989) . Exposure reconstruction is a step in that direction (Georgopoulos et al., 1994), although it is not always well statistically founded. A major problem, however, resides in accounting fully for the 
uncertainties stemming from unknown time-varying exposures. The impact of particular functional form for the time evolution of exposure has not yet been thoroughly studied and validated.

- Second, it is difficult to find a balance between "nice", powerful, physiological, complex PBPK models and simple, economical, easy to fit classical models when the number of individual studied gets large. This may well be a purely computational problem, but we are facing it. Minimal (or reduced) PBTK models may be promising tools to analyse data where many subjects are involved. In these models, some of the compartments (e.g., poorly and well-perfused tissues) have been lumped together. Reducing the number of compartments can be a way to simplify the model while partly preserving physiological realism. In the case of benzene, we showed that a reduced threecompartment semi-PBTK model can be a good alternative to a standard five-compartment PBTK model (Woodruff et al., 1992) . Thomaseth and Salvan (1998) considered a minimal physiological model to describe long-term kinetics of tetrachlorodibenzo- $p$-dioxin in human tissues, using data from a cohort of 359 subjects. This approach also needs to be further validated, but we feel that, as any modelling exercise, the development of a TK model should be essentially guided by the question raised and the data available to answer it.

- Third, the unfamiliarity with the approach. Here the situation is definitely improving and, hopefully, articles like this one will contribute to a better appreciation of the approach.

\section{TOXICODYNAMICS}

All the above remarks apply equally well to TD modelling, even though populations approaches are less developed in that area than in TK modelling (mostly for historical reasons). Biomarkers of effects, for example, call for the same treatment as biomarkers of exposure. Dose-response models can easy replace TK models in the population framework. An interesting application, at the TK/TD interface, is found at the occasion of drug exposure assessment in preclinical toxicological studies. In small rodents, repeated sampling may alter the health of the animals and jeopardise toxicity evaluation. To circumvent this limitation it is possible to collect serial samples from satellite animals maintained in conditions similar to those of the main study animals but not monitored for toxicity. Main study animals can then be only sparsely sampled. As noted above, sparse sampling designs are best analysed in population framework. Burtin et al. (1996) evaluated this option and showed that the data, analysed with a classical TK population model, 
yielded adequate estimation of drug disposition in both groups. An interesting extension would be to combine PBTK and PD models to analyse biomarker/epidemiological data on the toxicity of chemicals.

\section{PERSPECTIVES}

This rapid survey of the applications of population modelling to toxicology would not be complete without a few words about its limitations. The foremost may well be about a basic assumption of these models. To what extent is it correct to assign to randomness what could be, in fact, determined by time varying factors or other causal effects? When inter-individual differences could be due to age, for example, wouldn't it be better to try to model age as an explanatory variable? A very deterministic vision of the world would assume that the population approach is just a temporary band-aid while waiting for better models. It is likely, however, that population models will play an increasingly important role in exploratory analyses of heterogeneity of response to toxic exposures. They can at least point to research directions about the determinants of important differences in kinetics and actions of toxicants.

Another set of question is posed by the consideration of fully stochastic models, such as probabilistic cancer models (Bois and Compton-Quintana, 1992; Sherman and Portier, 2000; Tan, 1991). Stochastic models can explain heterogeneity in response by the random evolution of a process, without appealing to the hypothetical variability of some parameters. They tend, however, to be very complex and difficult to fit to data. It would be very interesting to associate population and stochastic modelling, in the case of cancer, for example (where the respective roles of "bad luck" and genetic makeup are not easy to dissociate).

A current priority could be the development of friendly, fast, flexible and cheap software for Bayesian population modelling. It is currently difficult to quickly explore alternative TK/TD models if they include more than about 5 parameters. Computer power increases daily, but that provide only part of the solution to the problem. Life is also too hard for Bayesians, for they routinely need to define prior distributions. That is not always an easy task, even when it would seem obvious, for reasons of data accessibility. While it is well known that some TK parameters are likely to exhibit inter- or intraindividual variability in humans (or other species), the only values readily available are "reference" values for young Caucasian males. Prior information about population variability may eventually be found by going back to the original publications but even those often lack adequate statistical treatment. Data about the shape of the distributions are even harder to find. That situation really needs to be improved. 
Despite these limitations, experience has been sufficient to demonstrate the feasibility and usefulness of the population approach in toxicology. Its potentials are just awaiting fuller exploitation.

\section{REFERENCES}

Aarons, L., 1992. Population pharmacokinetics. Int. J. Clin. Pharmacol. Ther. Toxicol. 30, 520-522.

Atkinson, A.C., Chaloner, K., Herzberg, A.M., Juritz, J., 1993. Optimum experimental designs for properties of a compartmental model. Biometrics 49, 325-337.

Bailer, A., 1997. An introduction to the use of physiologically based pharmacokinetic models in risk assessment. Stat. Methods Med. Res. 6, 341-358.

Beal, S.L., Sheiner, L.B., 1980. The NONMEM system. Am. Statist. 34, 118-119.

Beal, S.L., Sheiner, L.B., 1982. Estimating population kinetics. CRC Crit. Rev. Biomed. Eng. 8, 195-222.

Beatty, D., Piegorsch, W., 1997. Optimal statistical design for toxicokinetic studies. Stat. Methods Med. Res. 6, 359-376.

Bernardo, J.M., Smith, A.F.M., 1994. Bayesian Theory. Wiley, New York.

Bois, F.Y., 1999. Analysis of PBPK models for risk characterization. Ann. NY Acad. Sci. 895, 317-337.

Bois, F.Y., 2000a. Statistical analysis of Clewell et al. PBPK model of trichloroethylene kinetics. Environ. Health Perspect. 108 (suppl. 2), 307-316.

Bois, F.Y., 2000b. Statistical analysis of Fisher et al. PBPK model of trichloroethylene kinetics. Environ. Health Perspect. 108 (suppl. 2), 275-282.

Bois, F.Y., Compton-Quintana, P.G.E., 1992. Sensitivity analysis of a new model of carcinogenesis. J. Theor. Biol. 159, 361-375.

Bois, F.Y., Gelman, A., Jiang, J., Maszle, D., Zeise, L., Alexeef, G., 1996a. Population toxicokinetics of tetrachloroethylene. Arch. Toxicol. 70, 347-355.

Bois, F.Y., Jackson, E.T., Pekari, K., Smith, M.T., 1996b. Population toxicokinetics of benzene. Environ. Health Perspect. 104, 1405-1411.

Bois, F.Y., Smith, T.J., Gelman, A., Chang, H.Y., Smith, A.E., 1999. Optimal design for a study of butadiene toxicokinetics in humans. Toxicol. Sci. 49, 213-24.

Burtin, P., Mentre, F., Vanbree, J., Steimer, J.L., 1996. Sparse sampling for assessment of drug exposure in toxicological studies. European Journal of Drug Metabolism and Pharmacokinetics 21, 105-111.

Dankovic, D.A., Bailer, A.J., 1994. The impact of exercise and intersubject variability on dose estimates for dichloromethane derived from a physiologically based pharmacokinetic model. Fund. Appl. Toxicol. 22, 20-25. 
Davidian, M., Gallant, A.R., 1992. Smooth nonparametric maximum likelihood estimation for population pharmacokinetics, with application to quinidine. J Pharmacokinet Biopharm 20, 529-56.

Davidian, M., Giltinian, D., 1995. Nonlinear Models for Repeated Measurements Data. Chapman \& Hall, New-York.

Droz, P.O., Wu, M.M., Cumberland, W.G., 1989. Variability in biological monitoring of organic solvent exposure - II. Application of a population physiological model. Brit. J. Ind. Med. 46, 547-558.

Gargas, M.L., Medinsky, M.A., Andersen, M.E., 1995. Pharmacokinetic modeling approaches for describing the uptake, systemic distribution, and disposition of inhaled chemicals. Crit. Rev. Toxicol. 25, 237-254.

Gelman, A., Bois, F.Y., Jiang, J., 1996. Physiological pharmacokinetic analysis using population modeling and informative prior distributions. J. Am. Stat. Assoc. 91, 1400-1412.

Gelman, A., Rubin, D.B., 1996. Markov chain Monte Carlo methods in biostatistics. Stat Methods Med Res $5,339-55$.

Georgopoulos, P.G., Roy, A., Gallo, M.A., 1994. Reconstruction of short-term multi-route exposure to volatile organic compounds using physiologically based pharmacokinetic models. J. Exposure Anal. Environ. Epidemiol. 4, 309-328.

Gibaldi, M., Perrier, D., 1982. Pharmacokinetics (Second ed.). Dekker, New York.

Gilks, W.R., Richardson, S., Spiegelhalter, D.J., 1996. Markov Chain Monte Carlo in Practice. Chapman \& Hall, London.

Johanson, G., Jonsson, F., Bois, F.Y., 1999. Development of new technique for risk assessment using physiologically based toxicokinetic models. American Journal of Industrial Medecine suppl. 1, 101-103.

Kohn, M.C., 1995. Achieving credibility in risk assessment models. Toxicol. Lett. 79, 107-114.

Lunn, D.J., Aarons, L.J., 1997. Markov chain Monte Carlo techniques for studying interoccasion and intersubject variability: application to pharmacokinetic data. Appl. Statist. 46, 73-91.

Mallet, A., Mentre, F., Steimer, J.L., Lokiec, F., 1988. Nonparametric maximum likelihood estimation for population pharmacokinetics, with application to cyclosporine. J. Pharmacok. Biopharm. 16, 311-27.

McCann, K.S., 2000. The diversity-stability debate. Nature 405, 228-233.

Merlé, Y., Mentré, F., 1995. Bayesian design criteria: computation, comparison, and application to a pharmacokinetic and a pharmacodynamic model. J. Pharmacok. Biopharm. 23, 101-125.

Morris, J.B., Robinson, D.E., Vollmuth, T.A., Brown, R.P., Domeyer, B.E., 1996. A parallelogram approach for safety evaluation of ingested acetaldehyde. Regul Toxicol Pharmacol 24, 251-63.

Palmer, J.L., Müller, P., 1998. Bayesian optimal design in population models for haematologic data. Stat. Med. 17, 1613-1622. 
Patron-Bizet, F., Mentré, F., Genton, M., Thomas-Haimez, C., Maccario, J., 1998. Assessment of the global two-stage method to $\mathrm{EC}_{50}$ determination. J. Pharmacol. Toxicol. Methods 39, 103-108.

Racine-Poon, A., 1985. A Bayesian approach to nonlinear random effects models. Biometrics 41, 10151023.

Racine-Poon, A., Smith, A.F.M., 1990. Population models. In Statistical Methodology in the Pharmaceutical Sciences (D.A. Berry, Eds.), 139-162. Dekker, New York.

Rowe, W.D., 1994. Understanding uncertainty. Risk Anal. 14, 743-750.

Rowland, M., Tozer, T.N., 1989. Clinical Pharmacokinetics: Concepts and Applications (2nd ed.). Lea \& Febiger, Philadelphia.

Seber, G.A.F., Wild, C.J., 1989. Nonlinear Regression. John Wiley \& Sons, New York.

Sheiner, L.B., 1984. The population approach to pharmacokinetic data analysis: rationale and standard data analysis methods. Drug Metab. Rev. 15, 153-171.

Sheiner, L.B., Ludden, T.M., 1992. Population pharmacokinetics/dynamics. Annu. Rev. Pharmacol. Toxicol. $32,185-209$.

Sherman, C.D., Portier, C.J., 2000. Calculation of the cumulative distribution function of the time to a small observable tumor. Bull. Math. Biol. 62, 229-240.

Stirling, A., 1999. On science and precaution in the management of technological risk — Final report and summary of the study conducted under the auspices of an ESTO project on technological risk and the management of uncertainty. SPRU, University of Sussex.

Tan, W.Y., 1991. Stochastic Models of Carcinogenesis. Marcel Dekker, New York.

Thomaseth, K., Salvan, A., 1998. Estimation of occupational exposure to 2,3,7,8-tetrachlorodibenzo-pdioxin using a minimal physiologic toxicokinetic model. Environ Health Perspect 106 (suppl. 2), 743-53.

Vozeh, S., Steimer, J.L., Rowland, M., Morselli, P., Mentre, F., Balant, L.P., Aarons, L., 1996. The use of population pharmacokinetics in drug development. Clin. Pharmacokinet. 30, 81-93.

Wakefield, J.C., 1994. An expected loss approach to the design of dosage regimens via sampling-based methods. The Statistician 43, 13-29.

Wakefield, J.C., 1996. The Bayesian analysis of population pharmacokinetic models. J. Am. Stat. Assoc. 91, $62-75$.

Wakefield, J.C., Smith, A.F.M., Racine-Poon, A., Gelfand, A.E., 1994. Bayesian analysis of linear and nonlinear population models by using the Gibbs sampler. Appl. Statist. - J. Roy. Statist. Soc. C 43, 201-221.

Woodruff, T.J., Bois, F.Y., Auslander, D., Spear, R.C., 1992. Structure and parameterization of pharmacokinetic models: their impact on model predictions. Risk Anal 12, 189-201. 
Yuh, L., Beal, S., Davidian, M., Harrison, F., Hester, A., Kowalski, K., Vonesh, E., Wolfinger, R., 1994. Population pharmacokinetic/pharmacodynamic methodology and applications: a bibliography. Biometrics $50,566-575$. 


\section{FIGURE CAPTIONS}

Figure 1: Graphical representation of a population model. At the individual level, exposure (E), time (t) and individual characteristics (parameters, $\boldsymbol{\theta}$ ) condition the observations (data, y). The relationships between $\mathbf{E}, \mathbf{t}, \boldsymbol{\theta}$ and $\mathbf{y}$ are described by a model, $f$. Individual characteristics are randomly distributed in the population. Population means and variances ( $\mu$ and $\Sigma^{2}$ ) describe the shape of that distribution. Measurement errors, modelling errors, and intra-individual variability are

lumped in a common variance term $\sigma^{2}$. Unknown quantities are in circles, known quantities in square boxes. 


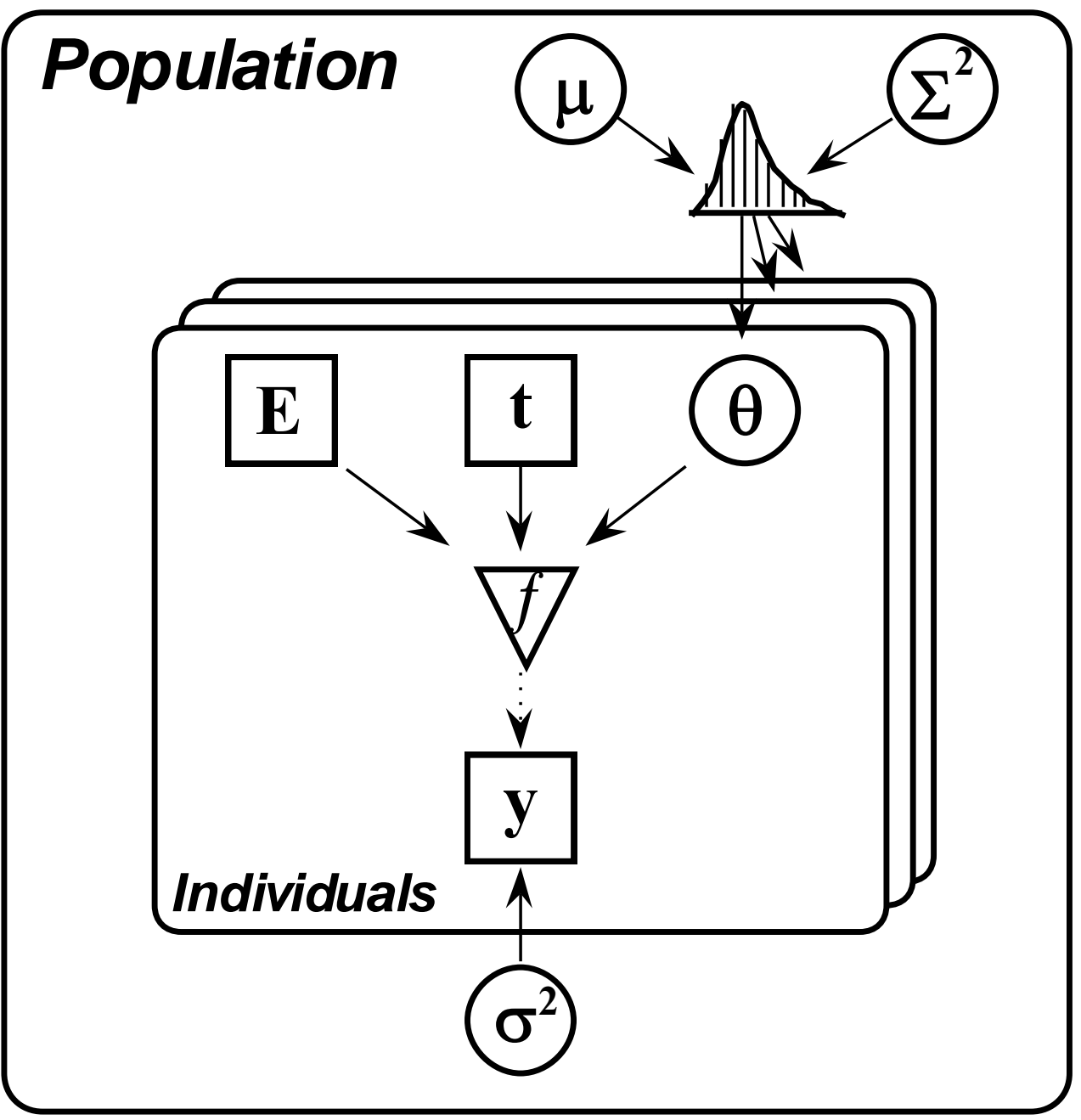

Figure 1: Graphical representation of a population model. At the individual level, exposure (E), time (t) and individual characteristics (parameters, $\theta$ ) condition the observations (data, y). The relationships between $\mathbf{E}, \mathbf{t}, \boldsymbol{\theta}$ and $\mathbf{y}$ are described by a model, $f$. Individual characteristics are randomly distributed in the population. Population means and variances ( $\boldsymbol{\mu}$ and $\sum^{2}$ ) describe the shape of that distribution. Measurement errors, modelling errors, and intra-individual variability are lumped in a common variance term $\sigma^{2}$. Unknown quantities are in circles, known quantities in square boxes. 